\title{
The effect of high positive-end expiratory pressure on elastance of respiratory system and ventilation distribution during robot-assisted laparoscopic radical prostatectomy
}

\author{
Shono A, Katayama N, Aoyama Y, Ozasa H, Nikai T, Saito Y. \\ Shimane University Faculty of Medicine, Dept of Anaesthesiology, Izumo City, Japan
}

\section{Background and Aim}

Pneumoperitoneum and steep Trendelenburg position during laparoscopic surgery increase elastance of the respiratory system and impair gas distribution in the lung, resulting in hypoxia.

In this study, we investigated the effect of high PEEP on elastance of respiratory system and regional ventilation distribution using Electrical Impedance Tomography (EIT) during robot-assisted laparoscopic prostatectomy (RALP).

\section{Methods}

Patients were ventilated with PCV mode, tidal volume was set at $6-8 \mathrm{ml} / \mathrm{kg} / \mathrm{PBW}$.

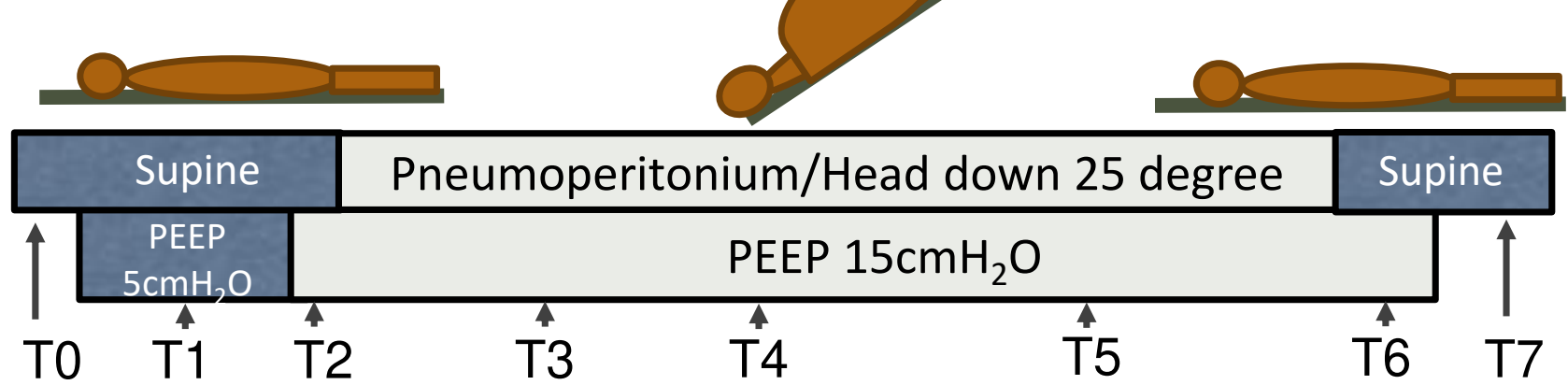

\begin{tabular}{|c|c|c|c|}
\hline \multicolumn{2}{|r|}{ Time points } & PEEP & \multirow{5}{*}{$\begin{array}{l}\text { Measurements } \\
\text { Respiratory parameters } \\
\checkmark \text { Tidal volume, } \mathrm{SpO}_{2}, \\
\text { Peak inspiratory } \\
\text { pressure, } \mathrm{FiO}_{2}, \\
\mathrm{PETCO}_{2}, \text { Respiratory } \\
\text { rate, } \mathrm{Cdyn}^{2}\end{array}$} \\
\hline T0 & before induction & & \\
\hline \multirow[t]{2}{*}{ T1 } & after induction & 5 & \\
\hline & Recruitment maneuver & & \\
\hline T2 & $\begin{array}{l}\text { before pneumoperitoneum } \\
\text { and head down }\end{array}$ & 15 & \\
\hline T3 & 20 min after head down & 15 & \multirow{5}{*}{$\begin{array}{l}\checkmark \text { Esophageal pressure } \\
\text { (Pes) } \\
\checkmark \text { Ventilation distribution } \\
\checkmark \text { Blood gas } \\
\text { Hemodynamics } \\
\checkmark \text { BP, HR } \\
\checkmark \text { Stroke volume variation } \\
\checkmark \text { Cardiac Index }\end{array}$} \\
\hline T4 & 60 min after head down & 15 & \\
\hline T5 & $120 \mathrm{~min}$ after head down & 15 & \\
\hline T6 & $\begin{array}{l}10 \text { min after cessation of } \\
\text { pneumoperitoneum }\end{array}$ & 15 & \\
\hline $\mathrm{T} 7$ & after extubation & & \\
\hline
\end{tabular}

* Calculation of Elastances: Chest wall (Ecw), Lung (EL)

Ers $=\frac{\text { Plateau pressure }- \text { PEEP }}{\text { Tidal volume }} \quad E L=$ Ers - Ecw

Ecw $=\frac{\text { Pes at end-inspiration }- \text { Pes at PEEP }}{\text { Tidal volume }}$

\section{- Electrical impedance tomography}

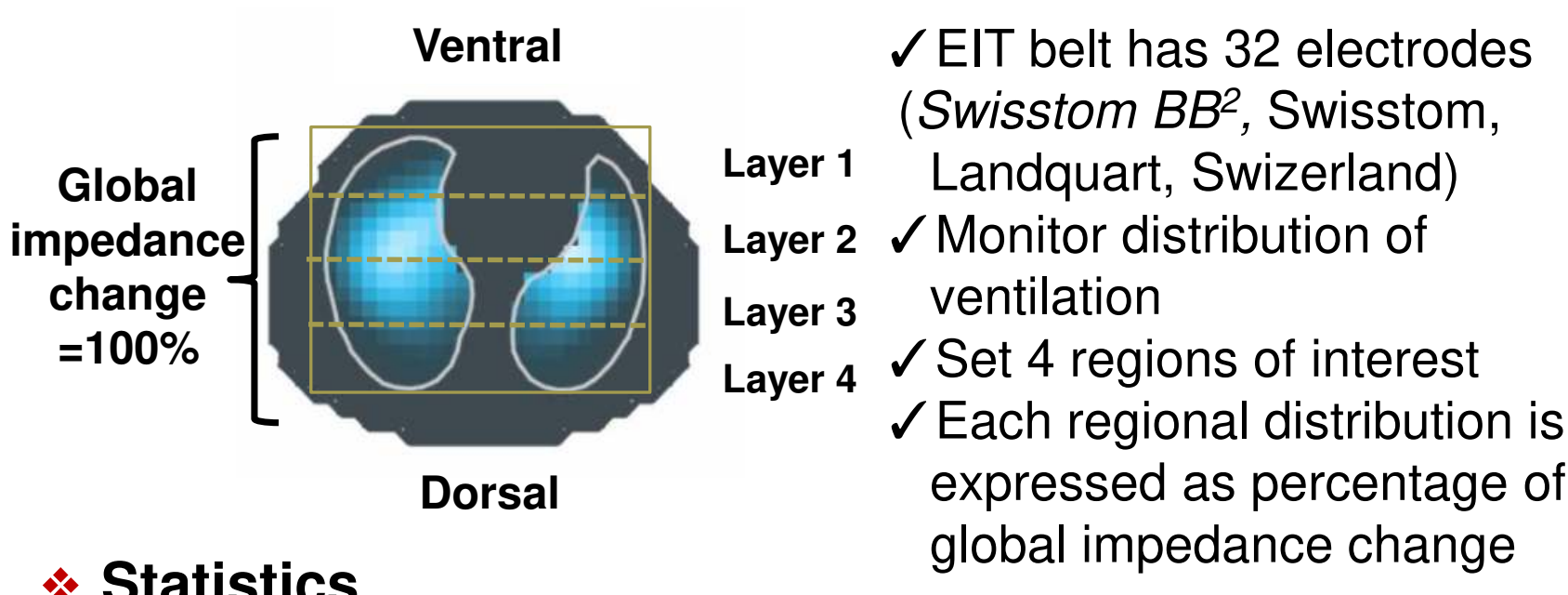

Data was analyzed by one-way ANOVA with repeated measures and Tukey test as post-hoc analysis.

\section{Results : Demographic data}

$$
\mathrm{n}=11
$$

Age (yr)

BMI $\left(\mathrm{kg} / \mathrm{m}^{2}\right)$

$\%$ Vital Capacity (\%)

FEV $1.0 \%(\%)$

Ope time (min)

Fluid balance (ml)

mean \pm SD

$67 \pm 2$

$24 \pm 2$

$100 \pm 4$

$76 \pm 2$

$408 \pm 28$

\section{Results : Change in Elastances}
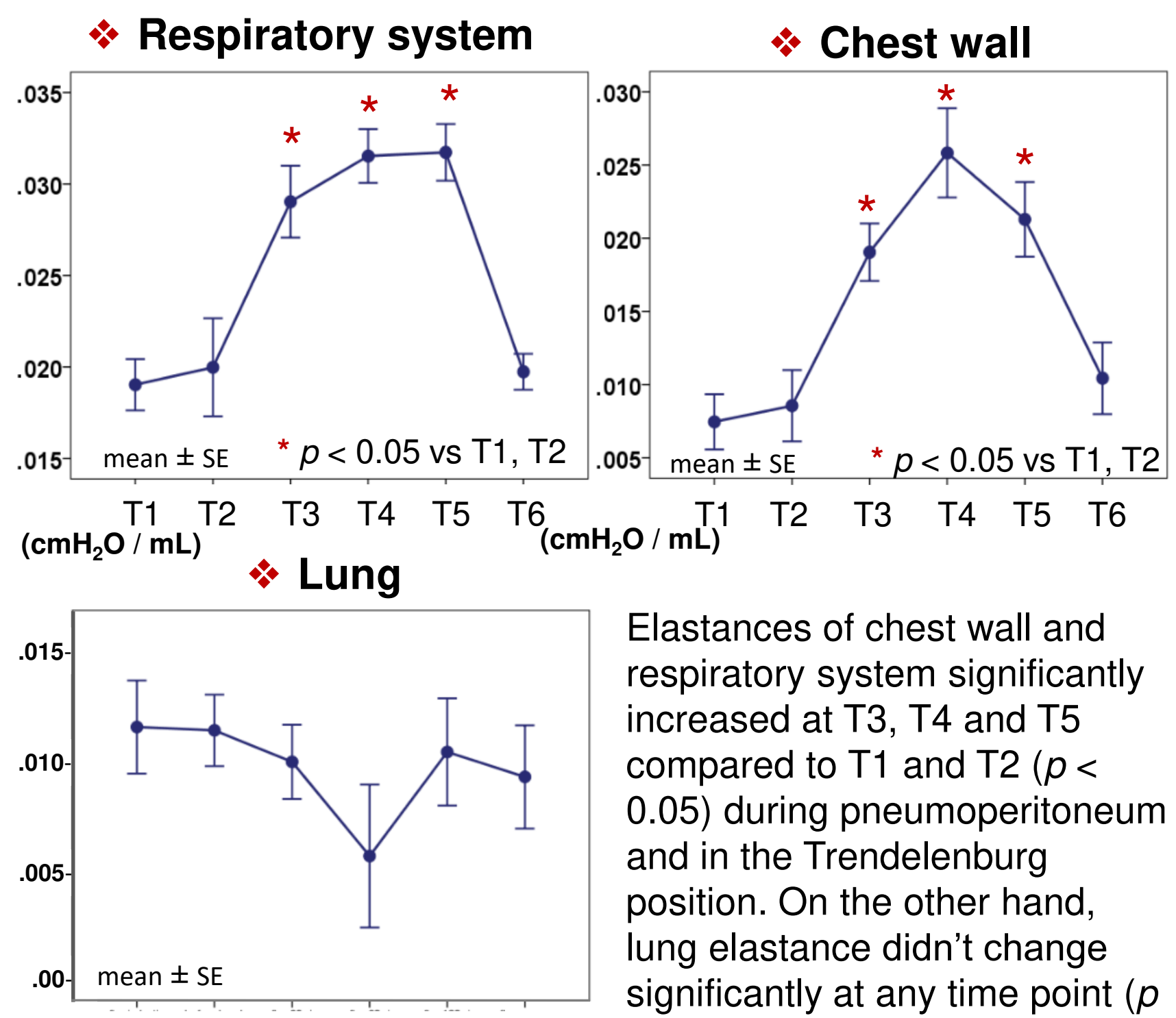
T1 $\begin{array}{llllll}\text { T2 } & \text { T3 } & \text { T4 } & \text { T5 } & \text { T6 } & =0.507)\end{array}$

$\left(\mathrm{cmH}_{2} \mathrm{O} / \mathrm{mL}\right)$

* P/F ratio

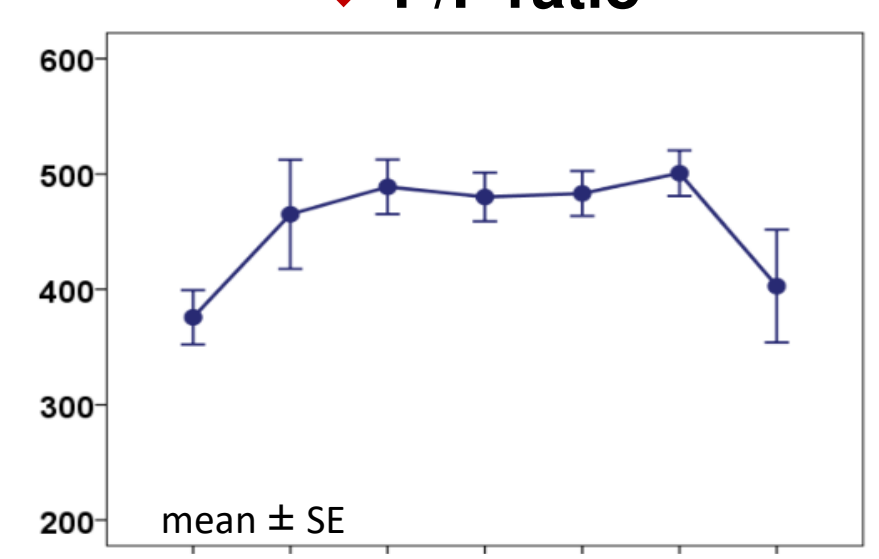

$\mathrm{P} / \mathrm{F}$ ratio at $\mathrm{T} 3, \mathrm{~T} 4$ and $\mathrm{T} 5$ were not lower than at $\mathrm{T} 1$ and $\mathrm{T} 2$.

Elastances of chest wall and respiratory system significantly increased at T3, T4 and T5 compared to T1 and T2 $(p<$ 0.05 ) during pneumoperitoneum and in the Trendelenburg position. On the other hand, lung elastance didn't change significantly at any time point $(p$

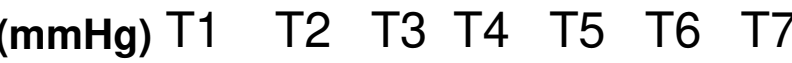

\section{Results : Change in distribution of ventilation}

\section{* Dorsal region : Layer 4}

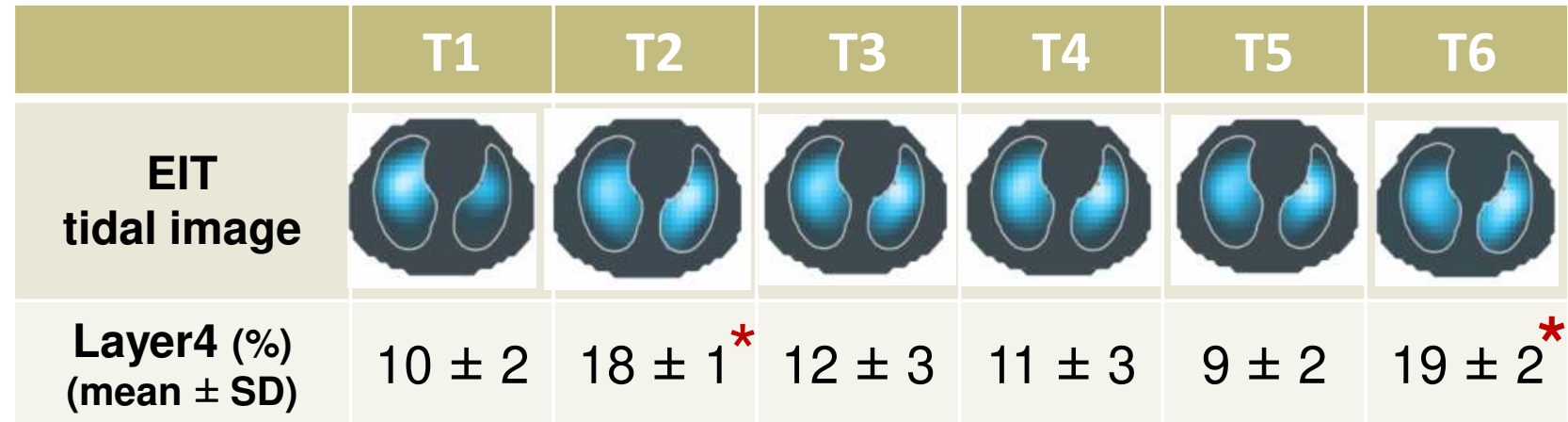

The ventilation distribution of the dependent part of the lung didn't decreased significantly at the Trendelenburg position compared to at $\mathrm{T} 1 .\left({ }^{*} p<0.05\right.$ vs T1)

\section{Discussion}

Elastance of chest wall increased due to pneumoperitoneum and Trendelenburg position. However, lung compliance was kept constant because lung elastance did not change during surgery. Dorsal ventilation during surgery monitored by EIT showed comparable distribution with at after induction. In addition, P/F ratio did not change through an entire study, indicating that lung collapse caused by pneumoperitoneum and Trendelenburg position might be prevented by applying high PEEP.

\section{Conclusions}

Lung elastance would not increase and dorsal ventilation could be maintained when high PEEP is applied before the establishment of pneumoperitoneum and Trendelenburg position. 